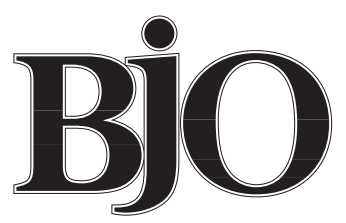

British Journal of Ophthalmology

\title{
Editorials
}

\section{Endophthalmitis following cataract extraction: the need for a systematic review of the literature}

Although endophthalmitis following cataract extraction is a rare complication (only $0.17 \%$ in the study by Norregaard et al presented in this issue of the $B \mathcal{O}$ (p 102)), endophthalmitis is important because frequently it has a significant effect on visual function.

Outcomes of cataract surgery, including endophthalmitis, have been the subject of intense investigation in recent years, in part because cataract extraction is the most commonly performed surgery in adults in most developed nations. In response to significant regional variation in cataract surgery rates across the USA, the Cataract Patient Outcome Research Team (PORT), funded by the Agency for Health Care Policy and Research (AHCPR) in the USA, was established to assess variations in short term and long term outcomes and costs for treatment of cataracts. ${ }^{1}$ Their review of Medicare records from 1984 revealed endophthalmitis rates within 1 year of inpatient cataract surgery of $0.17 \%$ for intracapsular cataract extraction and $0.12 \%$ for extracapsular extraction or phacoemulsification. ${ }^{2}$ The PORT team found that the endophthalmitis rate within 1 year following outpatient cataract surgery performed in 1986 and 1987 was $0.08 \%$. $^{3}$

A review of the medical literature to 1991, published by the AHCPR Cataract Management Guideline Panel, found that endophthalmitis rates following cataract extraction varied from 0 to $1.89 \%$ among 12 studies, but that the confidence limits around the rates were very large owing to the relatively small size of the populations studied and the rarity of the event. ${ }^{4}$ The panel recommended the initiation of well designed, well controlled, and possibly randomised studies of alternative techniques for cataract surgery to address questions of safety. Results from such studies have not been reported in the medical literature, but there have been a number of articles related to endophthalmitis. A Medline search of 5 years of the medical literature from 1992 to 1996 using the MESH headings 'endophthalmitis' and 'cataract extraction' uncovered 142 articles, 42 (30\%) of which were coded as case reports. The content of the articles varied and ranged from the case reports through to population based incidence estimates, risk factor analyses, and strategies for prophylaxis and treatment. In a review of endophthalmitis published in 1994, the authors concluded that multicentre clinical trials were necessary to assess the effectiveness of treatment regimens for endophthalmitis because the incidence is so low, but acknowledged that it was still doubtful that all possible variables could be accounted for. $^{5}$ They called for a national reporting system as the first step in identifying factors for future prospective studies, but noted the possible reticence of surgeon participation in a register of adverse outcomes because of the issue of potential litigation.

Although studies such as that reported by Norregaard and colleagues are very useful because they allow us to assess the effect of various healthcare systems on outcomes of cataract surgery, it is time for another systematic review of the literature in relation to endophthalmitis following cataract extraction. The Cochrane Collaboration prepares, maintains, and disseminates the results of systematic reviews of the effect of health care from reports in the medical literature (published and unpublished) to be used as the basis for evidence based decisions in health care. ${ }^{6}$ These systematic reviews are especially useful for rare outcomes, such as endophthalmitis, when no single study is likely to provide the answers necessary for rational evidence based clinical decision making because the incidence of endophthalmitis is so low. ${ }^{7}$ It is, nevertheless, very important because potentially it is one of the complications of cataract extraction that is the most visually disabling. With the overall success of cataract surgery very high, it is now important to elucidate the incidence, risk factors, and most effective treatment of the major complications of cataract extraction, including endophthalmitis. A systematic review of the extensive literature on endophthalmitis following cataract extraction could dramatically improve our understanding of this complication and save the vast resources that would be required to conduct a multicentred trial that is not assured of success. The results of a systematic review could also be used effectively to direct future prospective trials. The Cochrane Database of Systematic Reviews currently allows the rapid dissemination of results from completed reviews and in the future will allow the direct entry of data and immediate update of the register. The time has come for evidence based medicine, and management of endophthalmitis following cataract extraction is in need of an evidence base and systematic review.

CATHY MCCARTY

University of Melbourne, Department of Ophthalmology,

Royal Victorian Eye and Ear Hospital, 32 Gisborne Street,

East Melbourne, Victoria 3002, Australia 
1 Steinberg EP, Bergner M, Sommer A, Anderson GF, Bass EB, Canner J, et al. Variations in cataract management: patient and economic outcomes. Health Serv Res 1990;25:727-31.

2 Javitt JC, Vitale S, Canner JK, Street DA, Krakauer H, McBean AM, et al. National outcomes of cataract extraction. Endophthalmitis following inpatient surgery. Arch Ophthalmol 1991;109:1085-9.

3 Javitt JC, Street DA, Tielsch JM, Wang Q, Kolb MM, Schein O, et al. National outcomes of cataract extraction. Retinal detachment and endophthalmitis after outpatient cataract surgery. Ophthalmology 1994;101:100-6.

4 Cataract Management Guideline Panel. Management of functional impairment due to cataract in adults. Guideline Report, Number 4. Rockville, MD:
US Department of Health and Human Services, Public Health Service, Agency for Health Care Policy and Research. AHCPR Publication No Agency for Heath

5 Hughes DS, Hill RJ. Infectious endophthalmitis after cataract surgery. $B r \mathcal{F}$ Ophthalmol 1994;78:227-32.

6 Chalmers I, Dickerson K, Chalmers TC. Getting to grips with Archie Cochrane's agenda. BMF 1992;305:786-8.

7 Evidence-Based Medicine Working Group. Evidence-based medicine. A new approach to teaching the practice of medicine. $\mathscr{F} A M A 1992 ; 268$ : 2420-5.

\section{Macular holes viewed objectively}

Idiopathic full thickness macular holes (FTMH) are an important cause of poor vision in the elderly. Decreased visual function is mainly due to the absence of photoreceptors within the central retinal defect. Also, the adjacent cuff of subretinal fluid and secondary retinal elevation cause a relative scotoma surrounding the absolute central field defect.

The recent observation of Gass on the pathogenesis of FTMH $^{1}$ has sparked a number of investigations into new methods of assessment of FTMH. He described the vital importance of tangential vitreoretinal traction in the development and subsequent enlargement of FTMH and proposed a classification based on ophthalmoscopic signs. ${ }^{1}$ In stage 1 (impending) macular hole, the vitreoretinal traction causes foveal detachment which may progress to a small full thickness retinal defect (stage 2 FTMH). Stage 3 FTMH is usually larger and is associated with a cuff of subretinal fluid and focal vitreous detachment over the macula. In stage 4 there is complete vitreous separation. The interpretation of such signs depends on the diagnostic skills of the observer and is limited by the optical resolution of our current biomicroscopic methods. The need for improved diagnosis of such minute pathological changes has led to the development of new objective systems to record the anatomical and functional alterations both before and after treatment.

In most cases the diagnosis of FTMH can be made by biomicroscopy using a preset handheld lens or a Goldmann fundus contact lens. Patients with FTMH may also report a gap in a thin slit-beam projected on the macula $\left(\right.$ Watzke-Allen $\operatorname{sign}^{2}$ ) or the disappearance of a $50 \mu \mathrm{m}$ laser aiming beam shone in the centre of a macular hole. ${ }^{3}$ Such additional tests are useful in cases where the diagnosis of FTMH is in doubt. Indeed, in one series the diagnosis of macular pseudoholes by the initial ophthalmologist was correct in only $43 \% .^{4}$ An incorrect assessment may result in unnecessary surgery.

In an attempt to improve the diagnostic accuracy Tsujikawa and coworkers employed scanning laser ophthalmoscopic (SLO) microperimetry. In their study, published in this issue of the $B \mathcal{F O}(\mathrm{p} \mathrm{117})$, they examined a large series of patients with FTMH, macular pseudoholes, and impending (stage 1) macular holes. They correlated the presence of relative and absolute scotomata with the results of their ophthalmoscopic and peroperative findings. On preoperative biomicroscopy they were unable to classify nine of their 106 patients as FTMH or as a pseudohole. However, on SLO microperimetry four patients had a dense central scotoma; these patients were found to have FTMH at surgery. The remaining five eyes had only a relative scotoma, which appeared to correlate well with the peroperative diagnosis of macular pseudoholes. The high sensitivity as well as specificity of SLO microperimetry as demonstrated by Tsujikawa et al in this article is impressive and, if confirmed by further studies, may become part of the growing diagnostic armamentarium for researchers and surgeons in this field.

Since the first report of closure of macular holes with vitrectomy and gas tamponade $\mathrm{e}^{5}$ the reported surgical success has been steadily improving ${ }^{6-9}$ with or without the use of adjunctive substances such as transforming growth factor $\beta,{ }^{6}$ autologous serum, ${ }^{7}$ platelets, ${ }^{8}$ or thrombin. ${ }^{9}$ To date, the only explanation of the rather surprising visual improvement after the closure of FTMH has come from the histological examinations of postmortem eyes. ${ }^{10} 11$ These demonstrated the approximation of seemingly intact foveal photoreceptors to within $16 \mu \mathrm{m}$ from the central glial 'plug'. In vivo, a surgically closed macular hole and the surrounding retinal elevation may no longer be detectable ophthalmoscopically. However, such subtle changes in retinal thickness and elevation are not always easy to appreciate clinically and again in this issue (p 107) Hudson et al describe an objective method of documenting the changes in the retinal height before and after macular hole surgery using scanning laser tomography. In their study the analysis of digitised images of the retinal topography of the central $10^{\circ}$ and $20^{\circ}$ fields provided objective confirmation of the postoperative changes in retinal height in three of the four eyes they studied. Preoperatively, these three eyes had relatively large holes (stages 3 and 4 ) where the surrounding subretinal fluid may be quite marked. However, no such differences were found for the remaining eye which had a smaller (stage 2) FTMH presumably with less subretinal fluid associated with it, thus making any postoperative changes in the retinal height more difficult to detect. Nevertheless, from these preliminary results it looks as though scanning laser tomography as described by Hudson et al may prove to be helpful to the clinician in accurately recording and possibly predicting the postoperative result.

There are already a number of imaging techniques which have proved to be useful both in research and clinical management of FTMH. Some are well established such as fluorescein angiography, ${ }^{12}$ high resolution ultrasonography, ${ }^{13}$ and scanning laser ophthalmoscopy. ${ }^{14}$ By employing autofluorescence imaging the confocal SLO also allows differentiation between FTMH and pseudoholes without the inherent risks of an intravenous injection of fluorescein..$^{15} \mathrm{New}$ on the market is optical coherent tomography (OCT) which employs high resolution optical sections of the retina and allows for the measurement of 